\title{
Stabilizing Effect of Prey Refuge and Predator's Interference on the Dynamics of Prey with Delayed Growth and Generalist Predator with Delayed Gestation
}

\author{
Debaldev Jana \\ Ecological Modelling Laboratory, Department of Zoology, Visva-Bharati University, Santiniketan 731 235, India \\ Correspondence should be addressed to Debaldev Jana; debaldevjana.jana@gmail.com
}

Received 7 February 2014; Accepted 11 April 2014; Published 30 April 2014

Academic Editor: Daniel I. Rubenstein

Copyright (C) 2014 Debaldev Jana. This is an open access article distributed under the Creative Commons Attribution License, which permits unrestricted use, distribution, and reproduction in any medium, provided the original work is properly cited.

In the present paper, I study a prey-predator model with multiple time delays where the predator population is regarded as generalist. For this regard, I consider a Holling-Tanner prey-predator system where a constant time delay is incorporated in the logistic growth of the prey to represent a delayed density dependent feedback mechanism and the second time delay is considered to account for the length of the gestation period of the predator. Predator's interference in predator-prey relationship provides better descriptions of predator's feeding over a range of prey-predator abundances, so the predator's functional response here is considered to be Type II ratio-dependent. In accordance with previous studies, it is observed that delay destabilizes the system, in general, and stability loss occurs via Hopf bifurcation. There exist critical values of delay parameters below which the coexistence equilibrium is stable and above which it is unstable. Hopf bifurcation occurs when delay parameters cross their critical values. When delay parameters are large enough than their critical values, the system exhibits chaotic behavior and this abnormal behavior may be controlled by refuge. Numerical computation is also performed to validate different theoretical results. Lyapunov exponent, recurrence plot, and power spectral density confirm the chaotic dynamical behaviors.

\section{Introduction}

In ecology, predation describes a biological interaction where a predator (an organism that is hunting) feeds on its prey (the organism that is attacked). Predators may or may not kill their prey prior to feeding on them, but the act of predation often results in the death of its prey and the eventual absorption of the prey's tissue through consumption. Predators can have profound impacts on the dynamics of their prey that depend on how predator consumption is affected by prey density (the predator's functional response). A generalist species is able to thrive in a wide variety of environmental conditions and can make use of a variety of different resources (e.g., a heterotroph with a varied diet). Omnivores are usually generalists. Herbivores are often specialists, but those that eat a variety of plants may be considered generalists. Consumption by a generalist predator is expected to depend on the densities of all its major prey species (its multispecies functional response, or MSFR), but most studies of generalists have focused on their functional response to only one prey species [1-4]. Recently, many authors have explored the dynamics of a class of the semiratio-dependent [5-8]/Holling-Tanner [9-12]/Leslie-Grower $[13,14]$ predator-prey systems with functional responses as follows:

$$
\begin{gathered}
\frac{d x}{d t}=r x\left(1-\frac{x}{k}\right)-x f(x, y), \\
\frac{d y}{d t}=s y\left(1-\frac{h y}{x}\right),
\end{gathered}
$$

where $x(t)$ and $y(t)$ are, respectively, the prey and predator densities at time $t$. The prey population is assumed to grow logistically to its carrying capacity $k$ with intrinsic growth rate $r$ in absence of predator. $\alpha$ is the maximal per capita prey consumption rate, and $a$ is the amount of prey necessary for 
the relative biomass growth rate of the predator to be half its maximum; the predator consumes the prey according to the functional response $f(x, y)$ and grows logistically with growth rate $s$ and carrying capacity $x(t) / h$ proportional to the population size of the prey (or prey abundance). $h$ is the measure of the food quality that the prey provides, which is converted to predator birth. For more background and biological adjustments of system (1), one can see $[7,15,16]$ and the references cited therein.

Predator's functional response $f(x, y)$ is considered as Type II ratio-dependent [17-25] because a ratio-dependent predator-prey model does not show the so called paradox of enrichment [17, 26, 27] and biological control paradox [28]. The simplification of the food web structure due to predator interference allows qualitative predictions concerning the response of a food web to an external perturbation $[25,29-$ 32]. Ginzburg and Akcakaya [33] demonstrated a positive relationship between the abundances of all trophic levels and concluded that the ratio-dependent models provide a better representation of predator-prey interactions than prey dependent models. Predator's functional response, defined as the amount of prey catch per predator per unit of time, is affected by the structure of prey habitat and predator's hunting ability [34, 35]. Anderson [36] and Johnson [37] showed that the dynamics of a local population largely depend upon attributes of the local habitats. In addition, a spatial refuge protects a constant proportion of prey from predation. Mite predator-prey interactions often exhibit spatial refugia which afford the prey some degree of protection from predation and reduce the chance of extinction due to predation. Maynard Smith [38] shows that the presence of a constant proportion refuge does not alter the dynamical stability of the neutrally stable Lotka-Volterra model, while a constant number refuge of any size replaces the neutrally stable behaviour with a stable equilibrium. Hassel [39] shows that adding a large refuge to a model, which in the absence of a refuge exhibits divergent oscillations, replaces the oscillatory behaviour with a stable equilibrium. These mathematical models and a number of experiments indicate that refugia have a stabilizing effect on predator-prey interactions, but, as Taylor [40] has mentioned, it would be an over simplification to assume this is always the case. Kar [41] and Jana [42] proposed and studied a predatorprey system where prey population is subjected to refuge from their predator population. These papers lead a model by incorporating a refuge protecting $m x$ of the prey, where $x$ is the density of prey population and $m \in[0,1)$ is constant measures of the degree or strength of prey refuge. This leaves $(1-m) x$ of the prey available to the predator, so the HollingTanner predator-prey model with ratio-dependent functional response with prey refuge is given by

$$
\begin{gathered}
\frac{d x}{d t}=r x\left(1-\frac{x}{k}\right)-\frac{\alpha(1-m) x y}{a y+(1-m) x}, \\
\frac{d y}{d t}=s y\left[1-\frac{h y}{(1-m) x}\right] .
\end{gathered}
$$

Delay is frequently used in a predator-prey model to make the model biologically more realistic. Recently, researchers are using more than one delay to study the effect of past history of the system populations [43-47]. In the second phase of this study, I consider two delays in the model system (2). One discrete delay $\tau_{1}$ is considered in the specific growth rate of prey to incorporate the effect of density dependence feedback mechanism which takes $\tau_{1}$ units of time to respond to changes in the prey population [48]. The second delay $\tau_{2}$ is incorporated in the negative feedback of the predator density [49-52]. I thus obtain the following multidelayed predatorprey model:

$$
\begin{gathered}
\frac{d x}{d t}=r x\left(1-\frac{x\left(t-\tau_{1}\right)}{k}\right)-\frac{\alpha(1-m) x y}{a y+(1-m) x}, \\
\frac{d y}{d t}=s y\left[1-\frac{h y\left(t-\tau_{2}\right)}{(1-m) x\left(t-\tau_{2}\right)}\right] .
\end{gathered}
$$

The model system (3) has to be studied with the following initial conditions:

$$
\begin{gathered}
x(\phi)=x_{0}>0, \quad y(\phi)=y_{0}>0 \\
\text { for } \phi \in\left[-\max \left\{\tau_{1}, \tau_{2}\right\}, 0\right] .
\end{gathered}
$$

The organization of the paper is as follows. Section 2 deals with the analysis of the model system (3). Numerical studies are given in Section 3, and, finally, a brief discussion is presented in Section 4.

\section{Mathematical Results of System (3)}

The system (3) has only one interior equilibrium point given by $E^{*}\left(x^{*}, y^{*}\right)$, where $x^{*}=k(1-\alpha(1-m) / r(a+h))$ and $y^{*}=$ $(1-m) x^{*} / h$. The equilibrium point $E^{*}\left(x^{*}, y^{*}\right)$ is biologically feasible if $m>1-r(a+h) / \alpha, \alpha>r(a+h)$. Linearizing the system (3) at $\left(x^{*}, y^{*}\right)$, I get

$$
\begin{aligned}
& \frac{d x}{d t}=a_{11} x+a_{12} y+b_{11} x\left(t-\tau_{1}\right), \\
& \frac{d y}{d t}=c_{21} x\left(t-\tau_{2}\right)+c_{22} y\left(t-\tau_{2}\right),
\end{aligned}
$$

where

$$
\begin{gathered}
a_{11}=\frac{\alpha(1-m)^{2} x^{*} y^{*}}{\left\{a y^{*}+(1-m) x^{*}\right\}^{2}}, \\
a_{12}=-\frac{\alpha(1-m)^{2} x^{* 2}}{\left\{a y^{*}+(1-m) x^{*}\right\}^{2}}, \\
b_{11}=-\frac{r x^{*}}{k}, \\
c_{21}=\frac{s h(1-m) y^{* 2}}{\left\{(1-m) x^{*}\right\}^{2}}, \quad c_{22}=-\frac{s h y^{*}}{(1-m) x^{*}} .
\end{gathered}
$$

The corresponding characteristic equation is given by

$$
\lambda^{2}+A \lambda+C_{1} \lambda e^{-\lambda \tau_{1}}+\left(C_{2} \lambda+D_{2}\right) e^{-\lambda \tau_{2}}+E e^{-\lambda\left(\tau_{1}+\tau_{2}\right)}=0,
$$


where

$$
\begin{gathered}
A=-a_{11}, \quad C_{1}=-b_{11}, \quad C_{2}=-c_{22}, \\
D_{2}=a_{11} c_{22}-a_{12} c_{21}, \quad E=b_{11} c_{22} .
\end{gathered}
$$

I now discuss the following cases.

Case $1\left(\tau_{1}=0=\tau_{2}\right)$. In this case, $(7)$ becomes

$$
\lambda^{2}+\left(A+C_{1}+C_{2}\right) \lambda+\left(D_{2}+E\right)=0 .
$$

All roots of (9) will have negative real parts if and only if

$\left(\mathrm{H}_{1}\right)$

$$
A+C_{1}+C_{2}>0, \quad D_{2}+E>0 .
$$

Theorem 1. The interior equilibrium $E^{*}\left(x^{*}, y^{*}\right)$ of the system (3) exists and becomes locally asymptotically stable in absence of delays if $m>1-r(a+h) / \alpha, \alpha>r(a+h)$ and $\left(H_{1}\right)$ hold.

Case $2\left(\tau_{1} \neq 0, \tau_{2}=0\right)$. In this case, the characteristic equation (7) becomes

$$
\lambda^{2}+\left(A+C_{2}\right) \lambda+D_{2}+\left(C_{1} \lambda+E\right) e^{-\lambda \tau_{1}}=0
$$

Let $i \omega(\omega>0)$ be a root of (11). Then I have

$$
\begin{gathered}
E \cos \omega \tau_{1}+C_{1} \omega \sin \omega \tau_{1}=\omega^{2}-D_{2}, \\
C_{1} \omega \cos \omega \tau_{1}-E \sin \omega \tau_{1}=-\left(A+C_{2}\right) \omega .
\end{gathered}
$$

This leads to

$$
\omega^{4}-\left[C_{1}^{2}-\left(A+C_{2}\right)^{2}+2 D_{2}\right] \omega^{2}+D_{2}^{2}-E^{2}=0 .
$$

It follows that (12) has no positive roots if the following conditions are satisfied:

$\left(\mathrm{H}_{2}\right)$

$$
\left(A+C_{2}\right)^{2}-C_{1}^{2}-2 D_{2}>0, \quad D_{2}^{2}-E^{2}>0 .
$$

Hence, all roots of (13) will have negative real parts when $\tau_{1} \in[0, \infty)$ if conditions of Theorem 1 and $\left(\mathrm{H}_{2}\right)$ are satisfied.

Let

$\left(\mathrm{H}_{3}\right)$

$$
D_{2}^{2}-E^{2}<0
$$

If Theorem 1 and $\left(\mathrm{H}_{3}\right)$ hold, then (13) has a unique positive root $\widetilde{\omega}_{0}^{2}$. Substituting $\omega_{0}^{2}$ into (12), I have

$$
\begin{aligned}
\widetilde{\tau}_{1_{p}}= & \frac{1}{\widetilde{\omega}_{0}} \cos ^{-1}\left[\frac{E\left(\widetilde{\omega}_{0}^{2}-D_{2}\right)-\left(A+C_{2}\right) C_{1} \widetilde{\omega}_{0}^{2}}{C_{1}^{2} \widetilde{\omega}_{0}^{2}+E^{2}}\right] \\
& +\frac{2 p \pi}{\widetilde{\omega}_{0}}, \quad p=0,1,2, \ldots,
\end{aligned}
$$

where $\widetilde{\omega}_{0}$ is the unique positive root of (13).
Let

$\left(\mathrm{H}_{4}\right)$

$$
\begin{gathered}
\left(A+C_{2}\right)^{2}-C_{1}^{2}-2 D_{2}<0, \quad D_{2}^{2}-E^{2}>0, \\
{\left[\left(A+C_{2}\right)^{2}-C_{1}^{2}-2 D_{2}\right]^{2}>4\left(D_{2}^{2}-E^{2}\right) .}
\end{gathered}
$$

If $\left(\mathrm{H}_{1}\right)$ and $\left(\mathrm{H}_{4}\right)$ hold, then (13) has two positive roots $\widetilde{\omega}_{+}^{2}$ and $\widetilde{\omega}_{-}^{2}$. Substituting $\widetilde{\omega}_{ \pm}$into (12), we obtain

$$
\begin{aligned}
\widetilde{\tau}_{1_{q}}^{ \pm}= & \frac{1}{\widetilde{\omega}_{ \pm}} \cos ^{-1}\left[\frac{E\left(\widetilde{\omega}_{ \pm}^{2}-D_{2}\right)-\left(A+C_{2}\right) C_{1} \widetilde{\omega}_{ \pm}^{2}}{C_{1}^{2} \widetilde{\omega}_{ \pm}^{2}+E^{2}}\right] \\
& +\frac{2 q \pi}{\widetilde{\omega}_{ \pm}}, \quad q=0,1,2, \ldots
\end{aligned}
$$

If $\lambda\left(\tau_{1}\right)$ is a root of (11) satisfying $\operatorname{Re}\left(\lambda\left(\tau_{1_{n}}\right)\right)=$ 0 (or $\operatorname{Re}\left(\lambda\left(\tau_{1_{q}}^{ \pm}\right)\right)=0$, resp.) and $\operatorname{Im}\left(\lambda\left(\tau_{1_{n}}\right)\right.$ ) = $\omega_{0}$ (or $\operatorname{Im}\left(\lambda\left(\tau_{1_{q}}^{ \pm}\right)\right)=\widetilde{\omega}_{ \pm}$, resp.), I obtain

$$
\begin{aligned}
& {\left[\frac{d}{d \tau_{1}}\left(\operatorname{Re}\left(\lambda\left(\tau_{1}\right)\right)\right)\right]_{\widetilde{\tau}_{1_{0}, \omega=\widetilde{\omega}_{0}}}} \\
& \quad=\frac{\widetilde{\omega}_{0}^{4}+E^{2}-D_{2}^{2}}{\widetilde{\omega}_{0}^{2}\left(C_{1}^{2} \widetilde{\omega}_{0}^{2}+E^{2}\right)}>\frac{\widetilde{\omega}_{0}^{2}}{C_{1}^{2} \widetilde{\omega}_{0}^{2}+E^{2}}>0,
\end{aligned}
$$

by hypothesis $\left(\mathrm{H}_{4}\right)$.

Similarly, I can show that

$$
\begin{aligned}
& {\left[\frac{d}{d \tau_{1}} \operatorname{Re}\left(\lambda\left(\tau_{1}\right)\right)\right]_{\tau_{1}=\widetilde{\tau}_{1 q}^{+}, \omega=\widetilde{\omega}_{+}}>0,} \\
& {\left[\frac{d}{d \tau_{1}} \operatorname{Re}\left(\lambda\left(\tau_{1}\right)\right)\right]_{\tau_{1}=\widetilde{\tau}_{1}^{-}, \omega=\widetilde{\omega}_{-}}<0 .}
\end{aligned}
$$

Theorem 2. Assume that $\tau_{1} \neq 0, \tau_{2}=0$ and conditions of Theorem 1 are satisfied, then the equilibrium $E^{*}\left(x^{*}, y^{*}\right)$ is locally asymptotically stable for $\tau_{1}<\widetilde{\tau}_{1_{0}}$ and unstable for $\tau_{1}>$ $\widetilde{\tau}_{1_{0}}$. Furthermore, the system (3) undergoes a Hopf bifurcation at $\left(x^{*}, y^{*}\right)$ when $\tau_{1}=\widetilde{\tau}_{1_{0}}$, where

$$
\widetilde{\tau}_{1_{0}}=\frac{1}{\widetilde{\omega}_{0}} \cos ^{-1}\left[\frac{E\left(\widetilde{\omega}_{0}^{2}-D_{2}\right)-\left(A+C_{2}\right) C_{1} \widetilde{\omega}_{0}^{2}}{C_{1}^{2} \widetilde{\omega}_{0}^{2}+E^{2}}\right] .
$$

Case $3\left(\tau_{1} \in\left(0, \widetilde{\tau}_{1_{0}}\right), \tau_{2} \neq 0\right)$. In this case, I allow a gestation time period for the predator and also a constant time delay affecting the density dependent growth rate of the prey. I fix $\tau_{1}$ at some value from its stability range $\left(0, \widetilde{\tau}_{1_{0}}\right)$ and regard $\tau_{2}$ as a free parameter. I also assume that the model parameters are such, that hypothesis $\left(\mathrm{H}_{1}\right)$ holds. Let $i \omega$ with $\omega>0$ be a root of (7). Then,

$$
\omega^{4}+\widetilde{a} \omega^{2}+2 \widetilde{b} \sin \left(\omega \tau_{1}\right)+2 \widetilde{c} \cos \left(\omega \tau_{1}\right)+\widetilde{d}=0,
$$

where

$$
\begin{gathered}
\tilde{a}=A^{2}+C_{1}^{2}-C_{2}^{2}, \quad \tilde{b}=\omega C_{2} E-\omega^{3} C_{1}, \\
\widetilde{c}=-D_{2} E-\omega^{2} D_{1}+\omega^{2} A C_{1}, \quad \widetilde{d}=-\left(D_{2}^{2}+E^{2}\right) .
\end{gathered}
$$




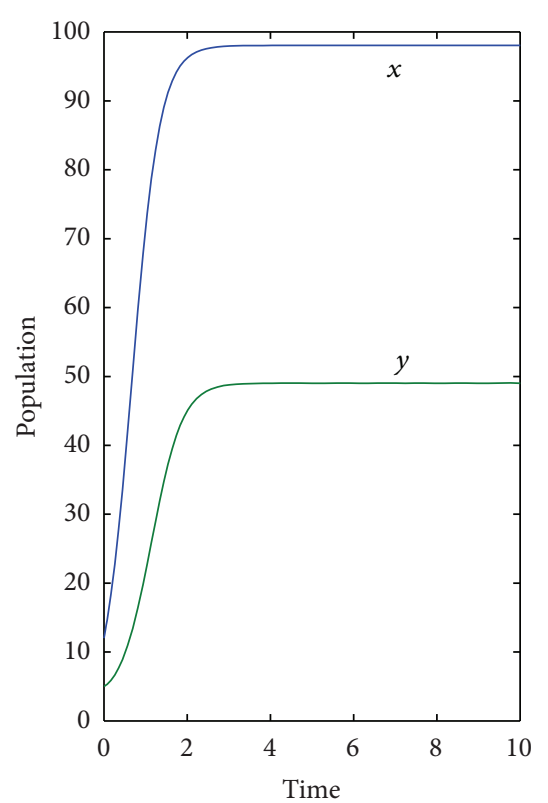

(a)

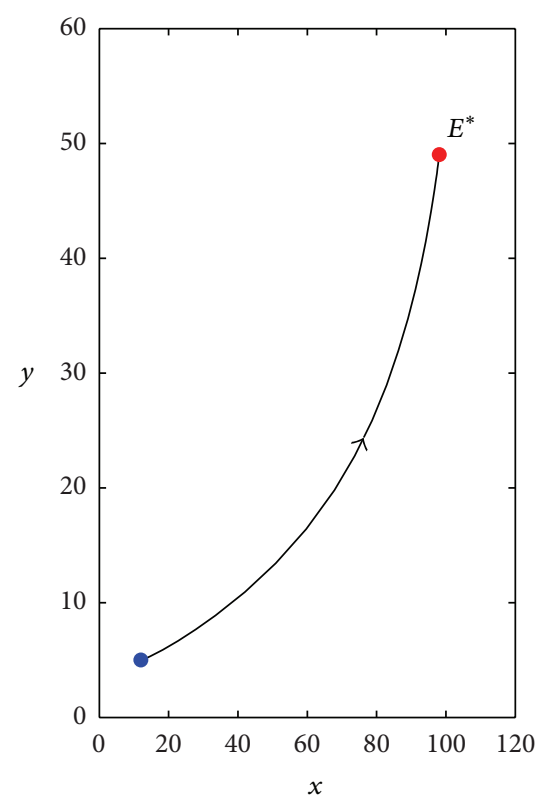

(b)

Figure 1: Time series (a) and phase-plane (b) of the system (2). This figure shows that the coexistence equilibrium is asymptotically stable.

Note that $\widetilde{d}$ is always negative. I define

$$
f(\omega)=\omega^{4}+\widetilde{a} \omega^{2}+2 \widetilde{b} \sin \left(\omega \tau_{1}\right)+2 \widetilde{c} \cos \left(\omega \tau_{1}\right)+\tilde{d}
$$

Then it is easy to verify that $f(0)<0$ and $f(\omega) \rightarrow \infty$, as $\omega \rightarrow \infty$. Thus, (22) has finitely many positive roots, denoted by $\widetilde{\omega}_{1}, \widetilde{\omega}_{2}, \ldots, \widetilde{\omega}_{k}$. For every fixed $\widetilde{\omega}_{s}$, where $s=1,2, \ldots, k$, there exists a sequence $\left\{\tilde{\tau}_{2_{s}}^{t} \mid t \in \mathbb{N}\right\}$, where

$$
\widetilde{\tau}_{2_{s}}^{t}=\frac{1}{\widetilde{\omega}_{s}} \cos ^{-1}\left(\frac{M_{2_{s}}}{N_{2_{s}}}\right)+\frac{2 s \pi}{\widetilde{\omega}_{s}}, \quad s=1,2, \ldots, k ; t \in \mathbb{N}
$$

with

$$
\begin{aligned}
M_{2_{s}} & =P_{1} S_{2}+P_{2} T_{2}+R_{2} T_{2} \cos \widetilde{\omega}_{s} \tau_{1}+R_{2} S_{2} \sin \widetilde{\omega}_{s} \tau_{1}, \\
N_{2_{s}} & =S_{2}^{2}+T_{2}^{2}, \quad P_{1}=-\widetilde{\omega}_{s}^{2}, \quad P_{2}=A \widetilde{\omega}_{s}, \\
R_{2} & =C_{1} \widetilde{\omega}_{s}, \\
S_{2} & =-\left(E \cos \widetilde{\omega}_{s} \tau_{1}+D_{2}\right), \\
T_{2} & =E \sin \widetilde{\omega}_{s} \tau_{1}-C_{2} \omega_{s}, \quad s=1,2, \ldots, k,
\end{aligned}
$$

such that (22) holds. Let $\tilde{\tau}_{2_{0}}=\min \left\{\tau_{2_{s}}^{t} \mid s=1,2, \ldots, k ; t=\right.$ $1,2, \ldots\}$. When $\tau_{2}=\widetilde{\tau}_{2_{0}},(7)$ has a pair of pure imaginary roots $\pm i \widetilde{\omega}_{1}$ for $\tau_{2} \in\left[0, \widetilde{\tau}_{2_{0}}\right)$. In the following, I assume that

$\left(\mathrm{H}_{5}\right)$

$$
\left[\frac{d}{d \tau_{2}}\left(\operatorname{Re} \lambda\left(\tau_{2}\right)\right)\right]_{\lambda=i \widetilde{\omega}_{0}} \neq 0
$$

Therefore, by the general Hopf bifurcation theorem of functional differential equations, see, for example, [53], I obtain the following result considering the change of stability of system (3).

Theorem 3. Suppose that the parameters in system (3) are such that hypotheses $\left(H_{1}\right)$ and $\left(H_{3}\right)$ hold true, and $\tau_{1} \epsilon$ $\left[0, \widetilde{\tau}_{1_{0}}\right)$. Then the coexistence equilibrium $E^{*}\left(x^{*}, y^{*}\right)$ is locally asymptotically stable when $\tau_{2} \in\left(0, \widetilde{\tau}_{2_{0}}\right)$, and it is unstable when $\tau_{2}>\widetilde{\tau}_{2_{0}}$. Moreover, Hopf bifurcation occurs when $\tau_{2}=\tilde{\tau}_{2_{0}}$.

Case $4\left(\tau_{1}=0, \tau_{2} \neq 0\right)$. For this choice of the delay parameters, I summarize my results in the following theorem. The proof follows similar arguments as the stability Theorem 2. in Case 2 above.

Theorem 4. Assume that $\tau_{1}=0, \tau_{2} \neq 0$ and the conditions in hypothesis $\left(H_{1}\right)$ hold true. Then the equilibrium $E^{*}\left(x^{*}, y^{*}\right)$ is locally asymptotically stable for $\tau_{2}<\tau_{2_{0}}$ and unstable for $\tau_{2}>$ $\tau_{2_{0}}$. Furthermore, the system (3) undergoes Hopf bifurcation when $\tau_{2}=\tau_{2_{0}}$, where

$$
\tau_{2_{0}}=\frac{1}{\omega_{0}} \cos ^{-1}\left(\frac{\left(D_{2}+E\right) \omega_{0}^{2}-\left(A+C_{1}\right) C_{2} \omega_{0}^{2}}{C_{2}^{2} \omega_{0}^{2}+\left(D_{2}+E\right)^{2}}\right),
$$

and $\omega_{0}$ is the unique positive root of the polynomial as follows:

$$
\omega^{4}-\left[C_{2}^{2}-\left(A+C_{1}\right)^{2}\right] \omega^{2}-\left(D_{2}+E\right)^{2}=0 .
$$

Case 5 ( $\tau_{1} \neq 0, \tau_{2} \neq 0$ and $\tau_{2}$ is within its stability range). This choice of parameter regime corresponds to the biologically interesting case, when the predator individuals have a fixed gestation period and the growth of the prey population is affected by delayed density dependent mechanisms. The proof of the main result follows similar lines to that in Case 


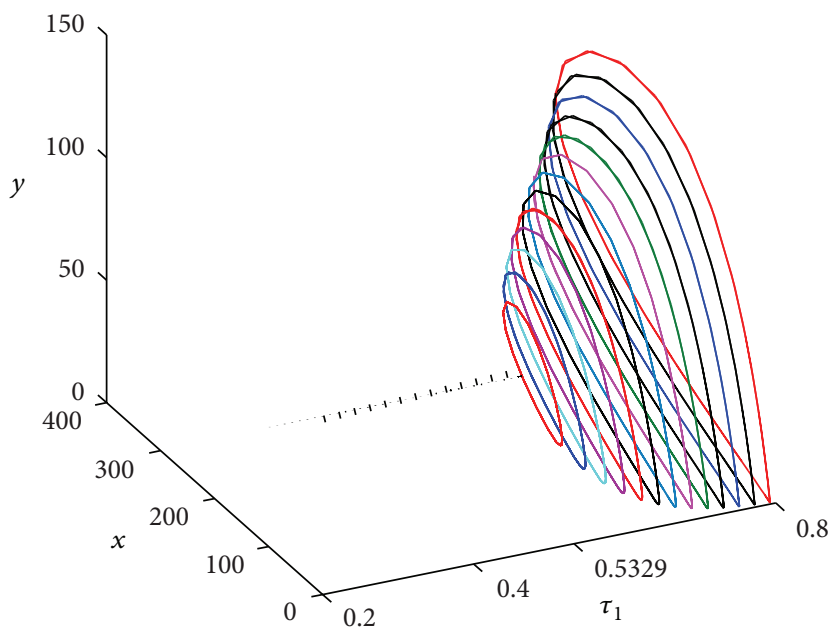

(a) $\tau_{2}=0$

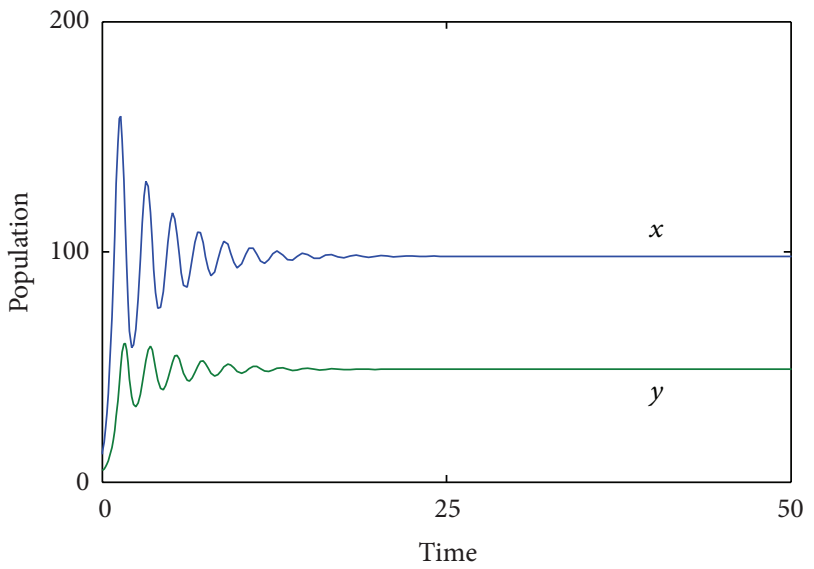

(b) $\tau_{1}=0.45$

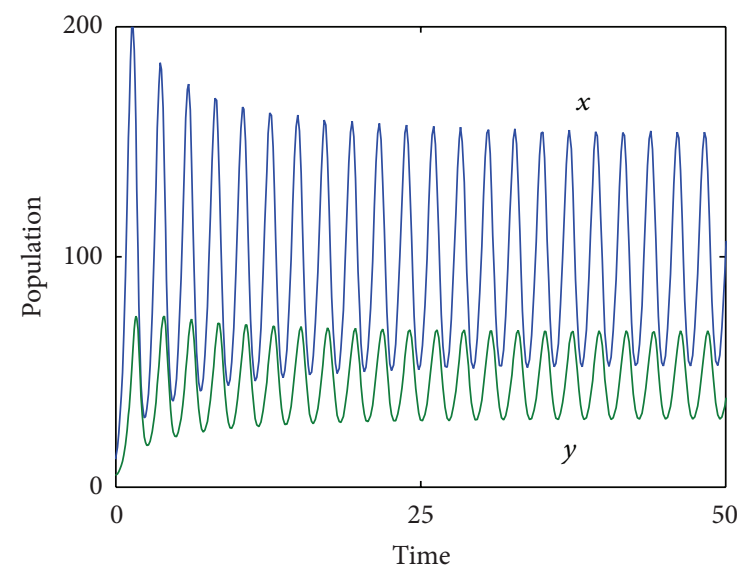

(c) $\tau_{1}=0.55$

FigURE 2: Bifurcation diagrams of the system (3) with respect to $\tau_{1}$ in Case 2 in the three-dimensional space $\left(\tau_{1}, x, y\right)$. (b) is the time series for $\left(\tau_{1}<\widetilde{\tau}_{1_{0}}\right)$ and (c) is the same for $\left(\tau_{1}>\widetilde{\tau}_{1_{0}}\right)$. These figures show that the coexistence equilibrium is stable for $\tau_{1}<0.5329$, unstable for $\tau_{1}>0.5329$, and a Hopf bifurcation exists at $\tau_{1}=0.5329$.

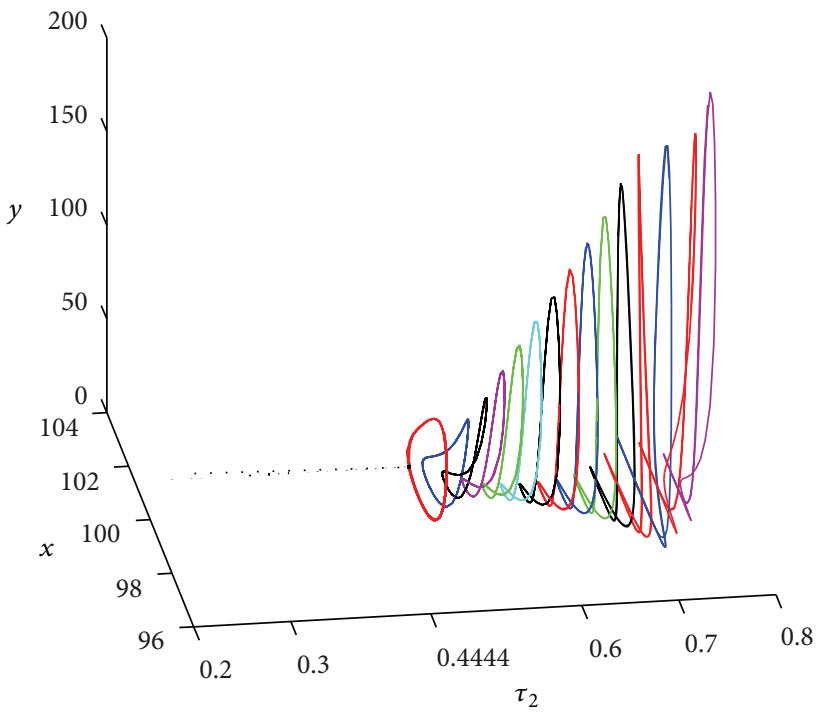

(a) $\tau_{1}=0.45$

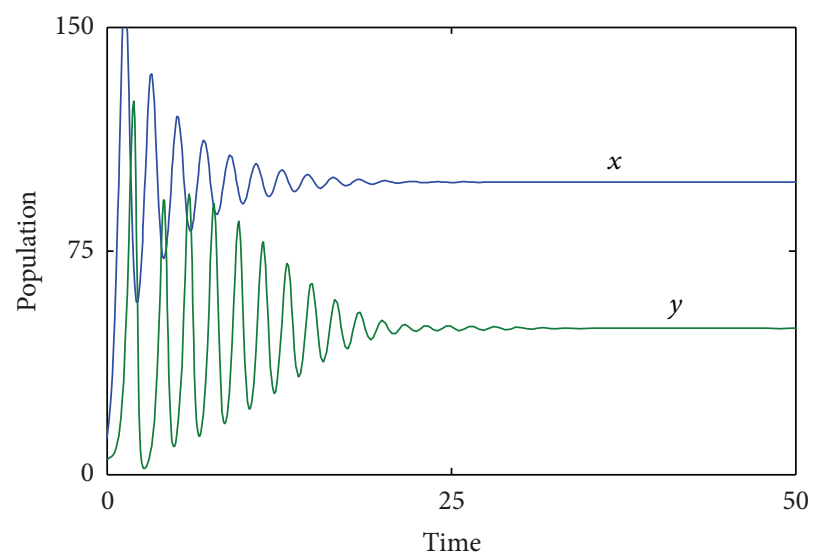

(b) $\tau_{2}=0.4$

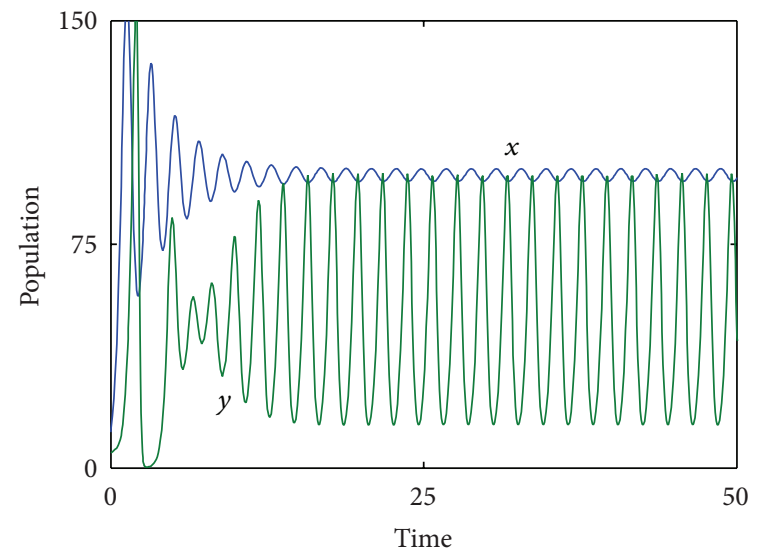

(c) $\tau_{2}=0.48$

FIgURE 3: Bifurcation diagram of the system (3) with respect to $\tau_{2}$ in Case 3. (a) is drawn in the three-dimensional space $\left(\tau_{2}, x, y\right)$. (b) and (c) are the time evolutions for $\tau_{2}=0.4<\widetilde{\tau}_{2_{0}}=0.4444$ (system is stable) and $\tau_{2}=0.48>\widetilde{\tau}_{2_{0}}=0.4444$ (system is unstable), respectively. A Hopf bifurcation exists at $\tau_{2}=0.4444$. Here, $\tau_{1}=$ $0.45 \in[0,0.5329)$. 


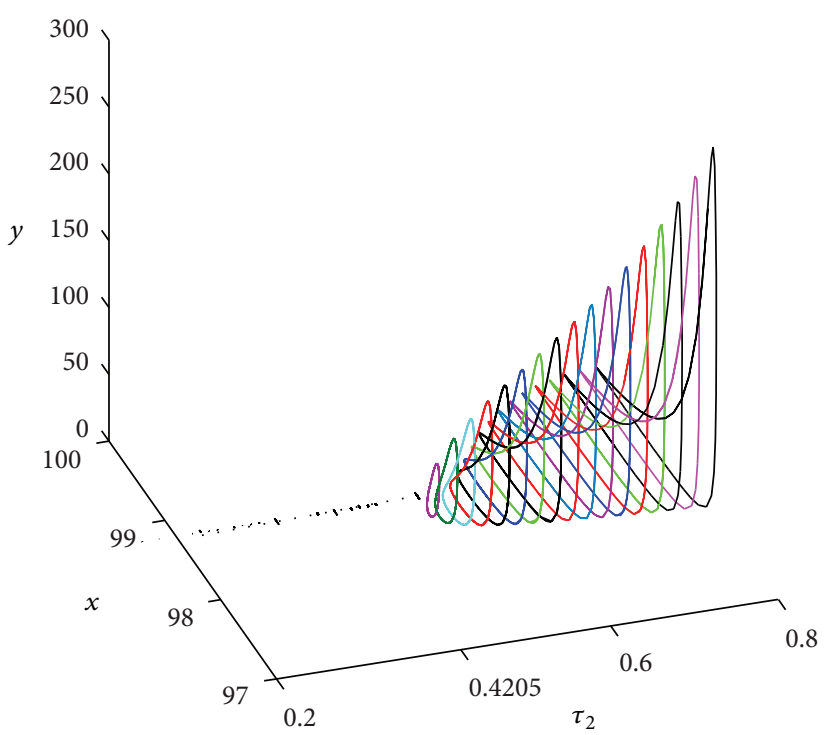

(a) $\tau_{1}=0$

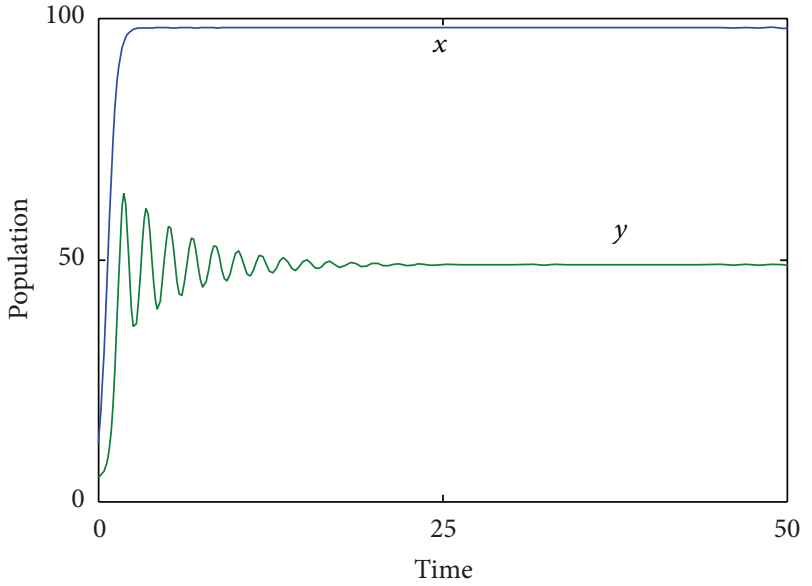

(b) $\tau_{2}=0.4$

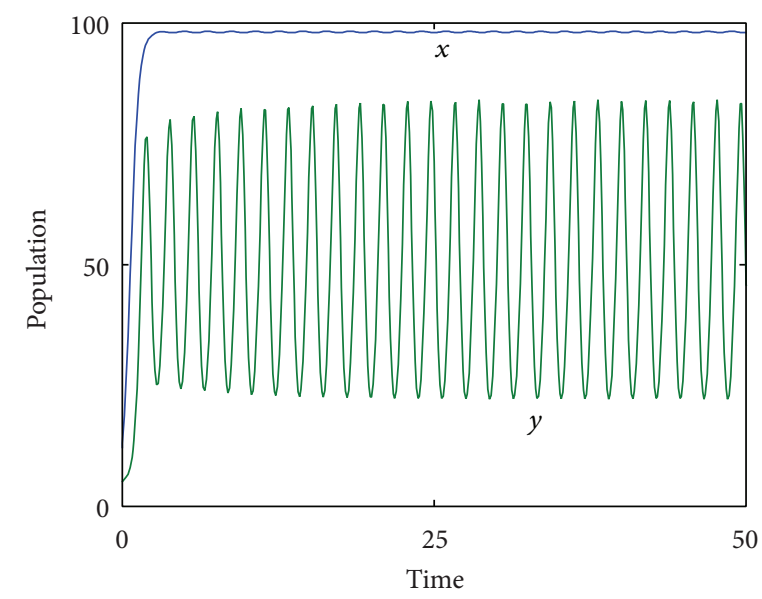

(c) $\tau_{2}=0.47$

FIGURE 4: Bifurcation diagrams of the system (3) with respect to $\tau_{2}$ in Case 4 in the three-dimensional space $\left(\tau_{2}, x, y\right)$. (b) is the time series for $\left(\tau_{2}<\tau_{2_{0}}\right)$ and (c) is the same for $\left(\tau_{2}>\tau_{2_{0}}\right)$. These figures show that the coexistence equilibrium is stable for $\tau_{2}<0.4205$, unstable for $\tau_{2}>0.4205$, and a Hopf bifurcation exists at $\tau_{2}=0.4205$.

3 and I only summarize the stability results in the following theorem.

Theorem 5. Assume that the model parameters are such that the hypotheses in $\left(H_{1}\right)$ hold true, and $\tau_{2} \in\left[0, \tau_{2_{0}}\right)$. Then the coexistence equilibrium $E^{*}\left(x^{*}, y^{*}\right)$ is asymptotically stable when $\tau_{1} \in\left[0, \tau_{1_{0}}\right)$, and it is unstable when $\tau_{1}>\tau_{1_{0}}$. Hopf bifurcation occurs when $\tau_{1}=\tau_{1_{0}}$, where $\tau_{1_{0}}=\min \left\{\tau_{1_{i}}^{j} \mid i=\right.$ $1,2, \ldots, k ; j \in \mathbb{N}\}$ and

$$
\tau_{1_{i}}^{j}=\left(\frac{1}{\omega_{i}}\right) \cos ^{-1}\left(\frac{M_{1_{i}}}{N_{1_{i}}}\right)+\frac{2 i \pi}{\omega_{i}}, \quad i=1,2, \ldots, k ; j \in \mathbb{N},
$$

with

$$
M_{1_{i}}=P_{1} S_{1}+P_{2} T_{1}+\left(Q_{1} S_{1}+R_{1} T_{1}\right) \cos \omega_{i} \tau_{2}
$$

$$
\begin{gathered}
\quad+\left(R_{1} S_{1}-Q_{1} T_{1}\right) \sin \omega_{i} \tau_{2}, \\
N_{1_{i}}=S_{1}^{2}+T_{1}^{2}, \quad P_{1}=-\omega_{i}^{2}, \quad P_{2}=A \omega_{i}, \\
Q_{1}=D_{2}, \quad R_{1}=C_{2} \omega_{i}, \\
S_{1}=-E \cos \omega_{i} \tau_{2}, \quad T_{1}=E \sin \left(\omega_{i} \tau_{2}\right)-C_{1} \omega_{i}, \\
i=1,2, \ldots, k .
\end{gathered}
$$

\section{Numerical Computations}

I perform numerical computations to observe various dynamics of the coexistence equilibrium for both the systems (2) and (3). I consider the fixed parameter values as $r=3, k=$ 100, $\alpha=1.0769, a=10, s=3.5, h=2$, and $m=0.35$. 


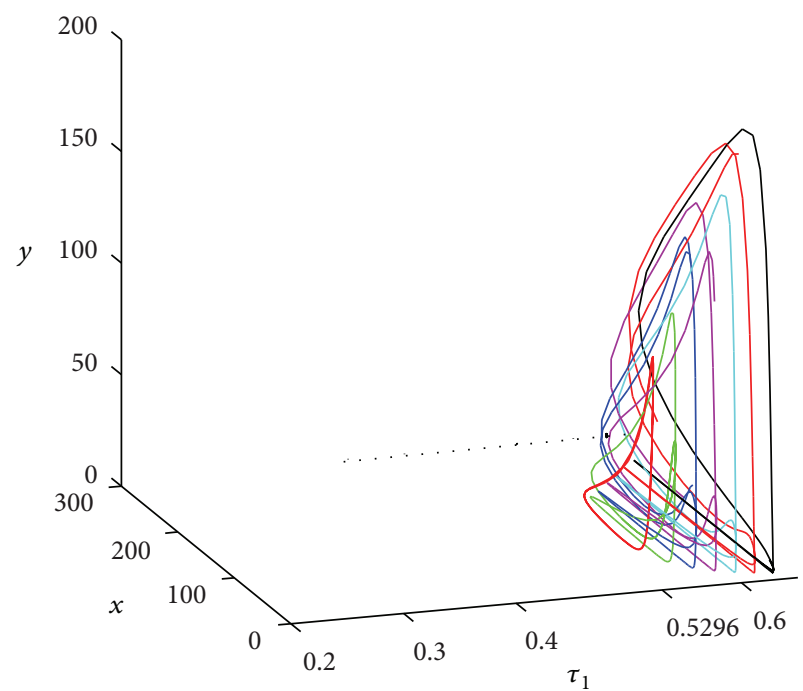

(a) $\tau_{2}=0.4$

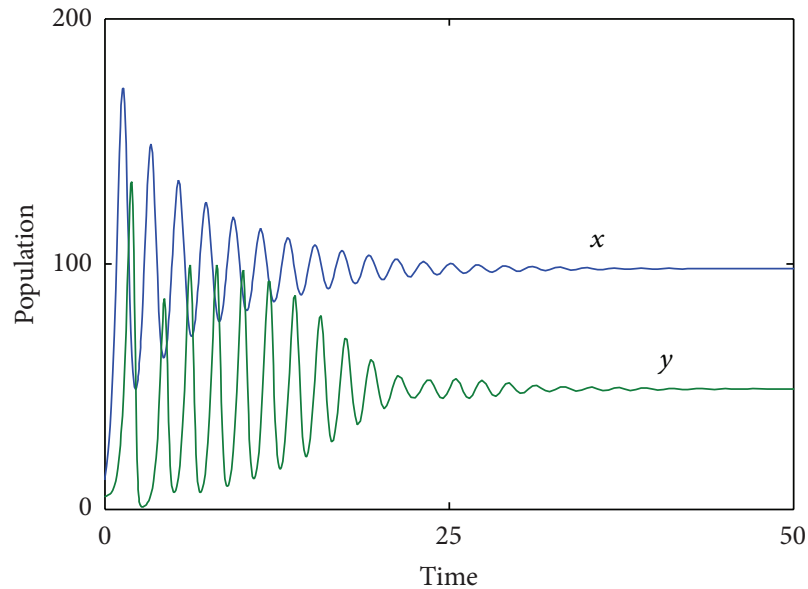

(b) $\tau_{1}=0.48$

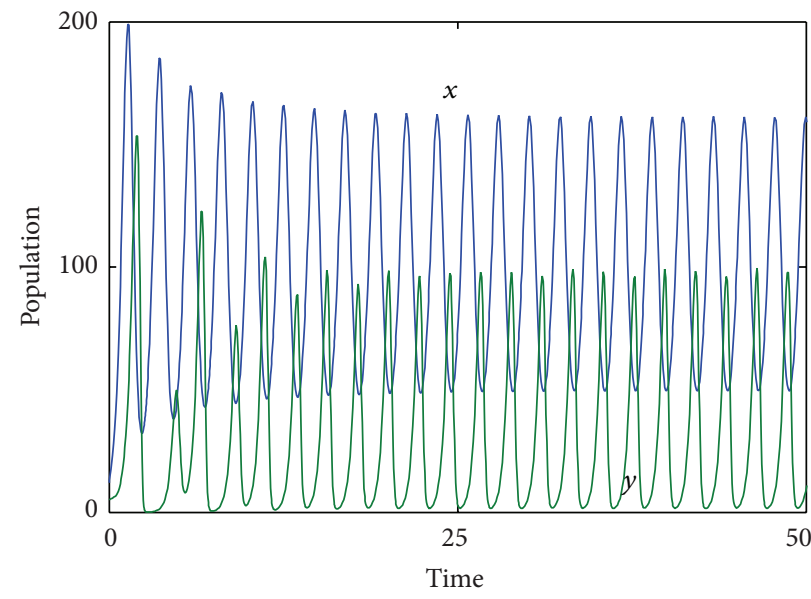

(c) $\tau_{1}=0.54$

Figure 5: Bifurcation diagram of the system (3) with respect to $\tau_{1}$ in Case 5. (a) is drawn in the three-dimensional space $\left(\tau_{1}, x, y\right)$. (b) and (c) are the time evolutions for $\tau_{1}=0.48<\tau_{1_{0}}=0.5296$ (system is stable) and $\tau_{1}=0.54>\tau_{1_{0}}=0.5296$ (system is unstable), respectively. A Hopf bifurcation exists at $\tau_{1}=0.5296$. Here, $\tau_{2}=0.4 \in[0,0.4205)$.

With these parameter values $x^{*}=98.0556, y^{*}=49.0278$. Here, $A+C_{1}+C_{2}=6.4319>0$ and $D_{2}+E=10.2618>0$, and initial value is considered as $(12,5)$ for each simulation. First I consider the nondelayed system (2). In this case, the system is asymptotically stable (Figure 1 ).

I now study the dynamic behavior of the delay-induced system (3). When $\tau_{1} \neq 0, \tau_{2}=0$ (Case 2), one can compute from Theorem 2 that $\widetilde{\omega}_{0}=2.9417, \tau_{1_{0}}=0.5329$, and $(A+$ $C 2)^{2}-C 1^{2}-2 D_{2}=3.5967>0 \& D_{2}^{2}-E^{2}=-106.003<0$. Therefore, the coexisting equilibrium $E^{*}\left(x^{*}, y^{*}\right)$ is asymptotically stable for $\tau_{1}=0.45<\widetilde{\tau}_{1_{0}}=0.5329$ (Figure 2(b)) and unstable for $\tau_{1}=0.55>\widetilde{\tau}_{1_{0}}=0.5329$ (Figure 2(c)). When $\tau_{1}=\widetilde{\tau}_{1_{0}}$, the system (3) undergoes a Hopf bifurcation at $E^{*}\left(x^{*}, y^{*}\right)$. System behaviors in Case 2 can be demonstrated more prominently if I plot the bifurcation diagram in the three-dimensional space $\left(\tau_{1}, x, y\right)$. Figure 2(a) shows that, when $\tau_{2}=0$, the coexistence equilibrium is stable for $\tau_{1}<$ $\widetilde{\tau}_{1_{0}}=0.5329$ but the instability sets in when $\tau_{1}>\widetilde{\tau}_{1_{0}}=0.5329$.

For Case 3, I take any value of $\tau_{1}$ from its stability range $[0,0.5329)$, say that $\tau_{1}=0.45$, and consider $\tau_{2}$ as a parameter. One can compute the value of $\widetilde{\omega}_{1}$ as 3.5345 and the corresponding critical value of $\tau_{2}$ as $\widetilde{\tau}_{2_{0}}=0.4444$. Thus, for fixed stable value of $\tau_{1}(=0.45)$, the system (3) exhibits stable behavior around $E^{*}\left(x^{*}, y^{*}\right)$ for $\tau_{2}<\widetilde{\tau}_{2_{0}}$ and unstable oscillatory behavior for $\tau_{2}>\widetilde{\tau}_{2_{0}}$ (Figure 3). A Hopf bifurcation occurs when $\tau_{2}$ takes the critical value $\widetilde{\tau}_{2_{0}}=$ 0.4444 .

In Case 4 , when $\tau_{1}=0, \tau_{2} \neq 0$, one can compute from Theorem $4, \omega_{0}=3.7359$ and $\tau_{2_{0}}=0.4205$. One can also verify that $\left(A+C_{1}\right)^{2}-C_{2}^{2}=-3.6537<0$ and $-\left(D_{2}+E\right)^{2}=$ $-105.3047<0$. Therefore, the coexisting equilibrium $E^{*}\left(x^{*}, y^{*}\right)$ is asymptotically stable for $\tau_{2}<\tau_{2_{0}}$, unstable for 


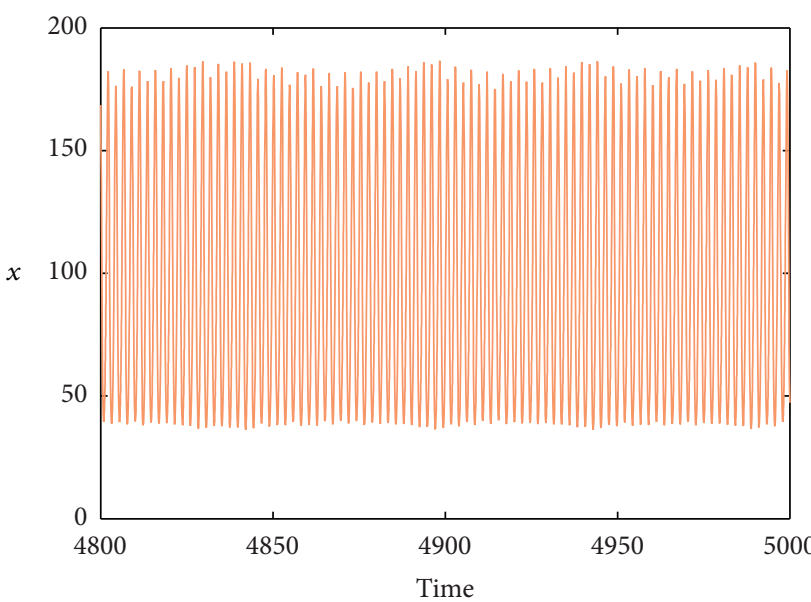

(a)

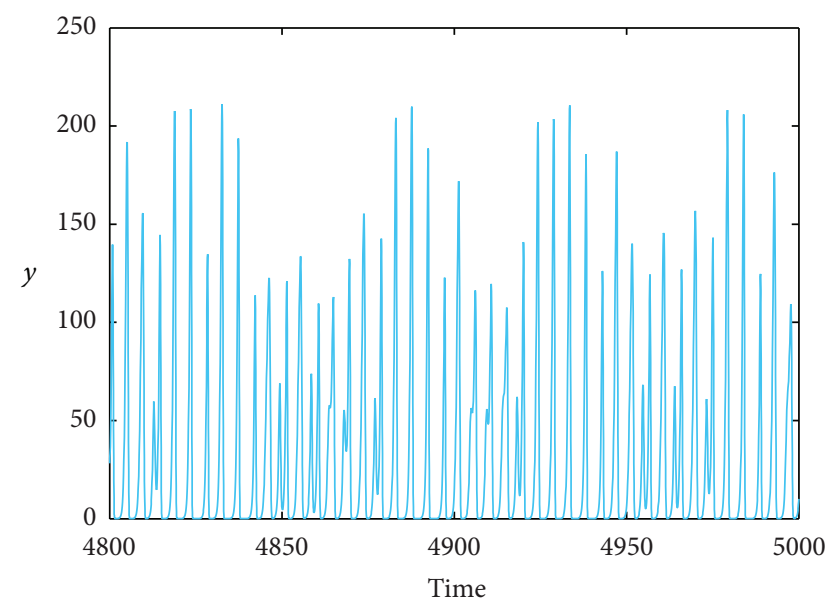

(b)

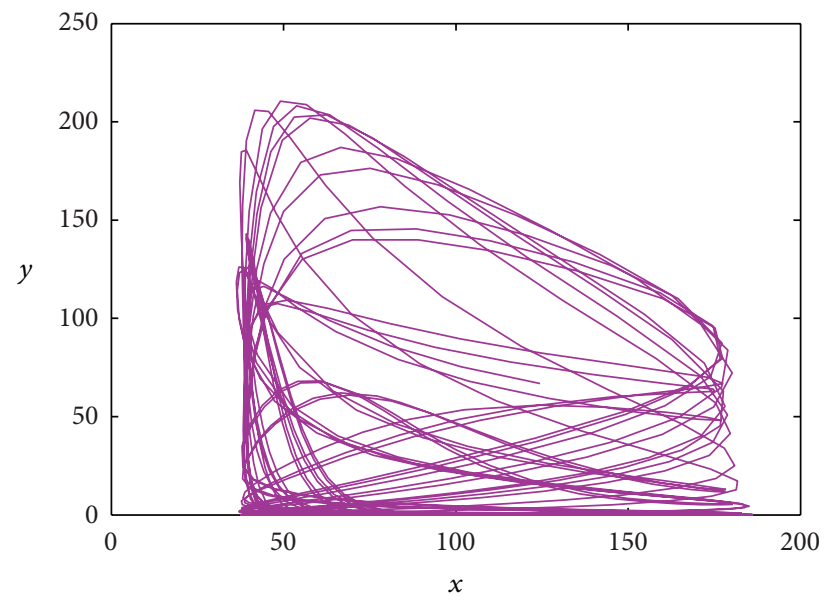

(c)

Figure 6: Time evolution (a)-(b) and phase-plane diagram (c) of the system (3) for $\tau_{1}=0.56$ and $\tau_{2}=0.535$. Here, the system (3) exhibits chaotic behavior. Other parameters are as in previous figures.

$\tau_{2}>\tau_{2_{0}}$, and when $\tau_{2}=\tau_{2_{0}}$, the system (3) undergoes a Hopf bifurcation at $E^{*}\left(x^{*}, y^{*}\right)$. These results can be observed from the bifurcation diagram Figure 4 .

In the last case (Case 5), I take any value of $\tau_{2}$ from its stability range $[0,0.4205)$, say that $\tau_{2}=0.4$, and consider $\tau_{1}$ as a parameter. As before, I calculate $\omega_{1}=2.96$ and the corresponding critical value $\tau_{1_{0}}=0.5296$. The bifurcation diagram Figure 5 demonstrates that the system (2) is locally asymptotically stable around $E^{*}\left(x^{*}, y^{*}\right)$ when $\tau_{1}<0.5296$ and unstable when $\tau_{1}>0.5296$. These results can be observed from the bifurcation diagram Figure 5.

3.1. Chaotic Dynamics. I have analytically studied the local behavior of the system (3) when the delay parameters are within or slightly above their critical values. One interesting topic in the delay-induced system is to study the dynamical behavior of the system when the delay parameters are far away from their critical values, or they assume large values. To observe the system dynamics, I consider $\tau_{1}=0.56$ and $\tau_{2}=0.535$, both are beyond their stability range, and then the system shows chaotic behavior (Figure 6).

In order to characterize the irregular behavior, I perform the standard numerical diagnostics, for example, Lyapunov exponent, sensitivity of the solutions to initial condition, power spectral density, and recurrence plot. Figure 7(a) shows the Lyapunov exponent of $y$ with respect to the delay $\tau_{2}$ in a smaller range $[0.45,0.539]$ for fixed $\tau_{1}$. Positive Lyapunov exponent $(\lambda)$ indicates that the system exhibits chaotic behavior $[42,54,55]$. Sensitivity of the system trajectories to the initial values is shown in Figure 7(b). To show this, I compute the error $\Delta y(t)=y_{1}(t)-y_{2}(t)$, where $y_{1}(0)=$ $(12,5)$ and $y_{2}(0)=(12.001,5)$. This phenomena ensures the chaotic nature of the system $[42,54,56]$. The power spectra of the predator population are presented in Figure 7(c). The irregular broad peaks of this figure are indicative of chaos and randomness $[42,54,57]$. The recurrence plot of the system for the parameter values as in Figure 6 is represented in Figure $7(\mathrm{~d})$. The random points on the time-time plane 


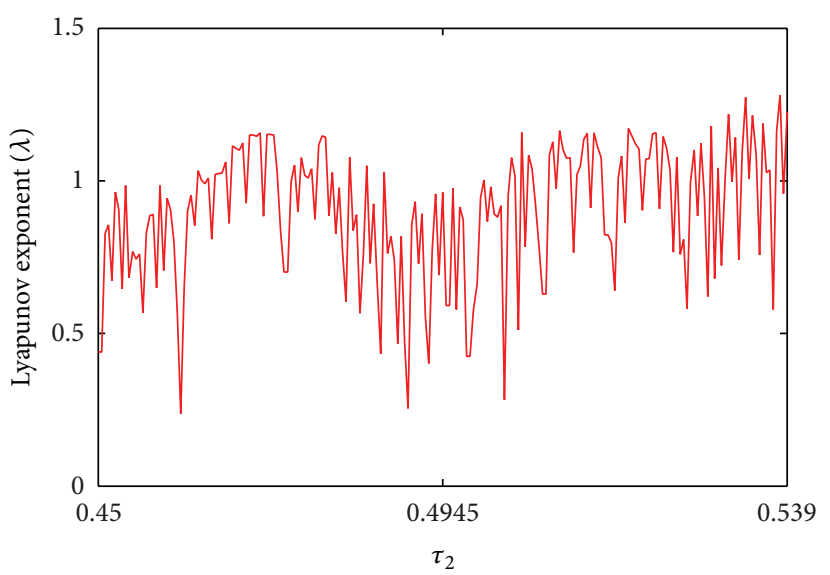

(a)

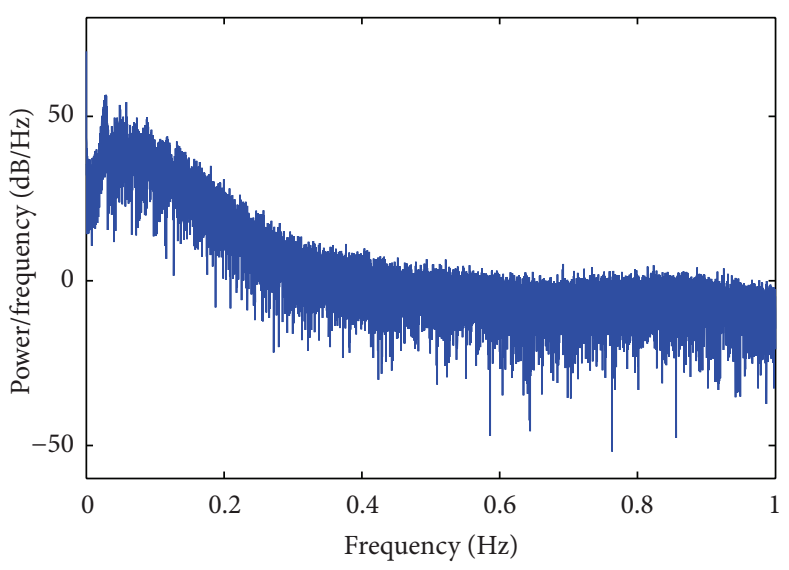

(c)

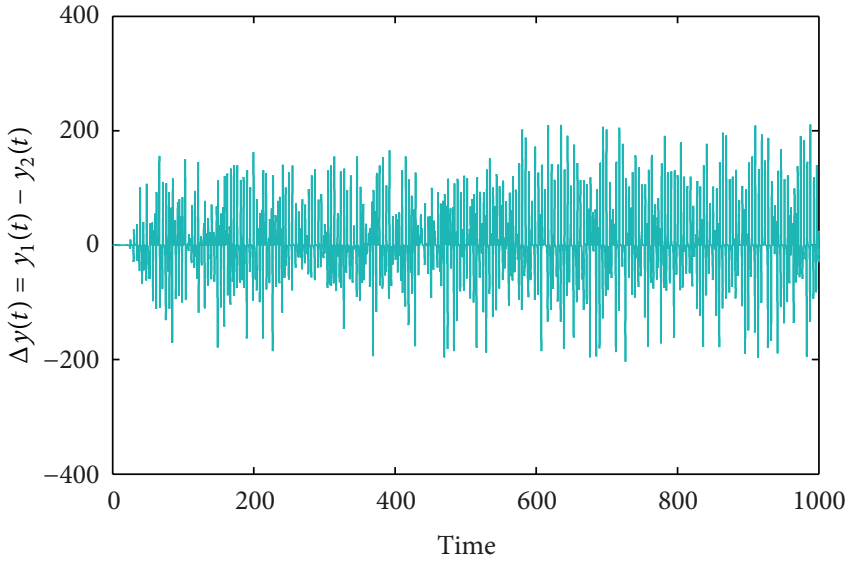

(b)

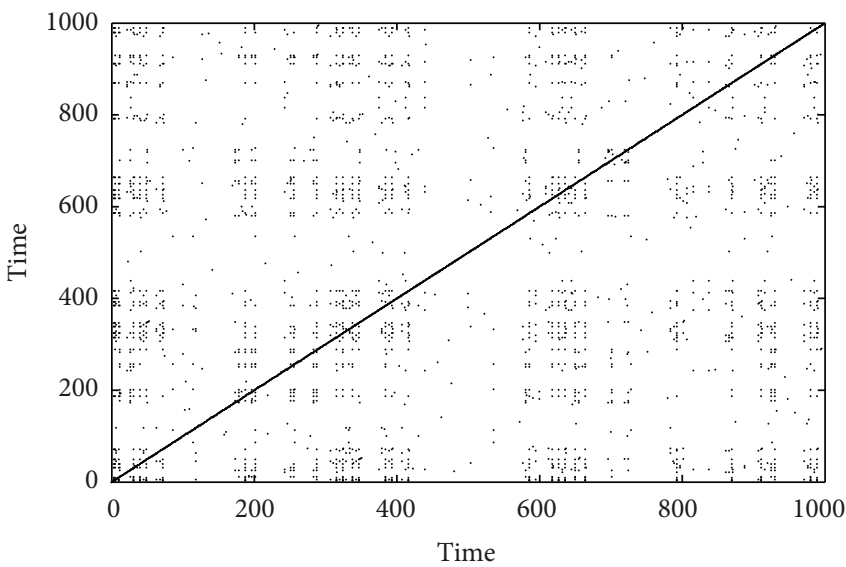

(d)

Figure 7: Standard diagnostic tests to detect chaos. (a) Lyapunov exponent, (b) sensitivity on the initial condition, (c) power spectral density, and (d) recurrence plot. Parameters are as in Figure 6.

ensure that more or less the same values of phase trajectories take place without any rhythm, indicating the chaotic nature of the system $[42,54,58]$.

\subsection{Biological Control of Chaotic Dynamics. Many ecological} situations show that their unstable or abnormal behavior may be controlled by any external perturbation $[14,21]$ or habitat structure [34, 36-40]. Recently Jana [42] shows that chaotic behavior of a discrete predator-prey system at low level of prey refuge must be controlled to its stable coexistence by increasing the degree of prey refuge. Parameter set of Figure 6 depicts the chaotic behavior of the system (3). Standing at this situation, if I increase the intensity of degree of prey refuge (from 0.35 to 0.6 ), then again the system (3) settles down to its stable coexistence (Figure 8).

\section{Discussion}

Effect of prey refuge has an extended impact on the predatorprey models. However, both field and laboratory experiments confirm that intensity of prey refuge reduces predation rates by decreasing encounter rates between predator and prey.
On the other hand, a predator-prey model becomes more realistic in presence of different delays which are unavoidable elements in physiological and ecological processes. In this paper, I have studied a multidelayed predator-prey model where the prey species is subject to partial refuge from predator population. Also I have considered here that the predator is a generalist type. A time delay is considered in the growth rate of the prey to represent density dependent feedback mechanism, and a second delay is introduced to account for the gestation time of the predator. The objective was to study the role of the effects of the interaction of multiple constant time delays and the prey refuge on the dynamical behavior of a predator-prey system. To have a better understanding of the complexities in natural systems, I can construct larger systems of differential equations containing more parameters. A second approach that is gaining prominence is the inclusion of time delay terms in the differential equations. Complex dynamical behaviour arises as a consequence of time delay in a biological system (with significant time delay) which may exhibit limited cycle oscillation and chaos. In case of the multidelayed system, I obtained sufficient conditions in terms of the system parameters for 


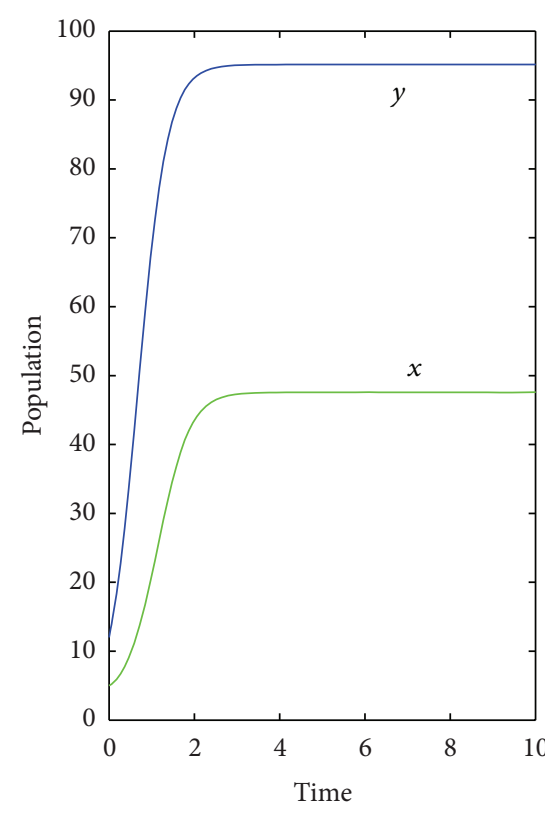

(a)

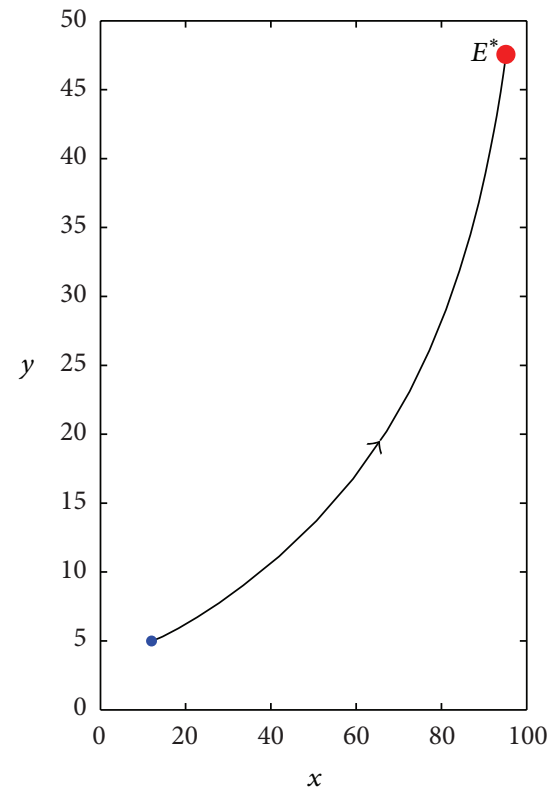

(b)

FIGURE 8: Biological control of chaos with increasing refuge $m=0.6$. Other parameters are as in Figure 6.

the stability of the coexistence equilibrium. I observed that the coexistence equilibrium is locally asymptotically stable if the delay parameters are lower than some critical values and it is unstable when the parameter values exceed these critical values. One important question in the multidelayed predatorprey system is to study the behavior of the system when the delay parameter is significantly large and hence it is far away from its critical value. As the estimated length of delays to preserve stability and the critical length of time delays for Hopf bifurcation are dependent on the system's parameters, it is possible to impose some control, which will prevent the possible abnormal oscillation in the population density. To explore the behavior of the system in this case, I performed extensive numerical simulations. My simulation results show that a refuged predator-prey system with multiple delays may exhibit different interesting (e.g., chaotic) behavior when the delay is large enough. Finally I showed that the chaotic characteristic of the multidelayed system was controlled by increasing the intensity of the prey refuge.

\section{Conflict of Interests}

The author declares that there is no conflict of interests regarding the publication of this paper.

\section{Acknowledgment}

This research is supported by UGC (Dr. D. S. Kothari Postdoctoral Fellowship), India; no. F.4-2/2006(BSR)/131004/2013(BSR).

\section{References}

[1] J. Matthiopoulos, K. Graham, S. Smout et al., "Sensitivity to assumptions in models of generalist predation on a cyclic prey," Ecology, vol. 88, no. 10, pp. 2576-2586, 2007.

[2] T. M. Temesgen, "Bifurcation analysis on the dynamics of a genralist predator-prey system," International Journal of Ecosystem, vol. 2, no. 3, pp. 38-43, 2012.

[3] Y. Kang and L. Wedekin, "Dynamics of a intraguild predation model with generalist or specialist predator," Journal of Mathematical Biology, vol. 67, no. 5, pp. 1227-1259, 2013.

[4] R. K. Upadhyay and S. R. K. Iyengar, Introduction to Mathematical Modelling and Chaotic Dynamics, Taylor and Francis, 2013.

[5] Q. Wang, M. Fan, and K. Wang, "Dynamics of a class of nonautonomous semi-ratio-dependent predator-prey systems with functional responses," Journal of Mathematical Analysis and Applications, vol. 278, no. 2, pp. 443-471, 2003.

[6] X.-X. Liu, "A note on periodic solutions for semi-ratio-dependent predator-prey systems," Applied Mathematics, vol. 25, no. 1, pp. 1-8, 2010.

[7] L. Hongying and W. Weiguo, "Dynamics of a delayed discrete semiratiodependent predator-prey system with Holling type IV functional response," Advances in Difference Equation, vol. 7, pp. 2-19, 2011.

[8] C. Lu and L. Zhang, "Permanence and global attractivity of a discrete semi-ratio dependent predator-prey system with Holling II type functional response," Journal of Applied Mathematics and Computing, vol. 33, no. 1-2, pp. 125-135, 2010.

[9] R. M. May, Stability and Complexity in Model Ecosystems, Princeton University Press, Princeton, NJ, USA, 1974.

[10] J. D. Murray, Mathematical Biology, Springer, Berlin, Germany, 1989. 
[11] P. A. Braza, "The bifurcation structure of the Holling-Tanner model for predator-prey interactions using two-timing," SIAM Journal on Applied Mathematics, vol. 63, no. 3, pp. 889-904, 2003.

[12] S.-B. Hsu and T.-W. Hwang, "Hopf bifurcation analysis for a predator-prey system of Holling and Leslie type," Taiwanese Journal of Mathematics, vol. 3, no. 1, pp. 35-53, 1999.

[13] H. C. Tsai and C. P. Ho, "Global stability for the lesliegower predator- prey system with time-delay and holling's type functional response," Tunghai Science, vol. 6, pp. 43-72, 2004.

[14] N. Zhang, F. Chen, Q. Su, and T. Wu, "Dynamic behaviors of a harvesting Leslie-Gower predator-prey model," Discrete Dynamics in Nature and Society, vol. 2011, Article ID 473949, 14 pages, 2011.

[15] J. T. Tanner, "The stability and the intrinsic growth rates of prey and predator populations," Ecology, vol. 56, pp. 855-867, 1975.

[16] S. B. Hsu and T. W. Hwang, "Uniqueness of limit cycles for a predator-prey system of Holling and Lesile type, Canad," Applied Mathematics Quarterly, vol. 6, pp. 91-117, 1998.

[17] Y. Kuang and E. Beretta, "Global qualitative analysis of a ratiodependent predator-prey system," Journal of Mathematical Biology, vol. 36, no. 4, pp. 389-406, 1998.

[18] A. Martin and S. Ruan, "Predator-prey models with delay and prey harvesting," Journal of Mathematical Biology, vol. 43, no. 3, pp. 247-267, 2001.

[19] C. Jost, O. Arino, and R. Arditi, "About deterministic extinction in ratio-dependent predator-prey models," Bulletin of Mathematical Biology, vol. 61, no. 1, pp. 19-32, 1999.

[20] D. Xiao and S. Ruan, "Global dynamics of a ratio-dependent predator-prey system," Journal of Mathematical Biology, vol. 43, no. 3, pp. 268-290, 2001.

[21] D. Jana, S. Chakraborty, and N. Bairagi, "Stability, nonlinear oscillations and bifurcation in a delay-induced predator-prey system with harvesting," Engineering Letters, vol. 20, no. 3, pp. 238-246, 2012.

[22] D. Xiaq and L. S. Jennings, "Bifurcations of a ratio-dependent predator-prey system with constant rate harvesting," SIAM Journal on Applied Mathematics, vol. 65, no. 3, pp. 737-753, 2005.

[23] D. Xiao, W. Li, and M. Han, "Dynamics in a ratio-dependent predator-prey model with predator harvesting," Journal of Mathematical Analysis and Applications, vol. 324, no. 1, pp. 1429, 2006.

[24] S. Chakraborty, S. Pal, and N. Bairagi, "Predator-prey interaction with harvesting: mathematical study with biological ramifications," Applied Mathematical Modelling, vol. 36, no. 9, pp. 4044-4059, 2012.

[25] H. R. Akcakaya, R. Arditi, and L. R. Ginzburg, "Ratio-dependent predation: an abstraction that works," Ecology, vol. 76, no. 3, pp. 995-1004, 1995.

[26] N. G. Hairston, F. E. Smith, and L. B. Slobodkin, "Community structure, population control and competition," American Naturalist, vol. 94, pp. 421-425, 1960.

[27] M. L. Rosenzweig, "Paradox of enrichment: destabilization of exploitation ecosystems in ecological time," Science, vol. 171, no. 3969, pp. 385-387, 1971.

[28] R. F. Luck, "Evaluation of natural enemies for biological control: a behavioral approach," Trends in Ecology and Evolution, vol. 5, no. 6, pp. 196-199, 1990.

[29] D. Kesh, D. Mukherjee, A. K. Sarkar, and A. B. Roy, "Ratio dependent predation: a bifurcation analysis," The Korean Journal of Computational \& Applied Mathematics, vol. 5, no. 2, pp. 295305, 1998.
[30] F. Bartumeus, D. Alonso, and J. Catalan, "Self-organized spatial structures in a ratio-dependent predator-prey model," Physica A: Statistical Mechanics and its Applications, vol. 295, no. 1-2, pp. 53-57, 2001.

[31] L. R. Ginzburg and R. X. J. Jensen, "From controversy to consensus: the indirect interference functional response," Verhandlungen des Internationalen Verein Limnologie, vol. 30, no. 2, pp. 297-301, 2008.

[32] C. Cosner, D. L. Deangelis, J. S. Ault, and D. B. Olson, "Effects of spatial grouping on the functional response of predators," Theoretical Population Biology, vol. 56, no. 1, pp. 65-75, 1999.

[33] L. R. Ginzburg and H. R. Akcakaya, "Consequences of ratiodependent predation for steady-state properties of ecosystems," Ecology, vol. 73, no. 5, pp. 1536-1543, 1992.

[34] D. Alstad, Basic Populas Models of Ecology, Prentice Hall, Upper Saddle River, NJ, USA, 2001.

[35] O. Anderson, "Optimal foraging by largemouth bass in structured environments," Ecology, vol. 65, no. 3, pp. 851-861, 1984.

[36] T. W. Anderson, "Predator responses, prey refuges, and densitydependent mortality of a marine fish," Ecology, vol. 82, no. 1, pp. 245-257, 2001.

[37] D. W. Johnson, "Predation, habitat complexity, and variation in density-dependent mortality of temperate reef fishes," Ecology, vol. 87, no. 5, pp. 1179-1188, 2006.

[38] J. Maynard Smith, Models in Ecology, Cambridge University Press, Cambridge, UK, 1974.

[39] M. P. Hassel, The Dynamics of Arthropod Predator-Prey Systems, Princeton University Press, Princeton, NJ, USA, 1978.

[40] R. J. Taylor, Predation, Chapman \& Hall, New York, NY, USA, 1984.

[41] T. K. Kar, "Stability analysis of a prey-predator model incorporating a prey refuge," Communications in Nonlinear Science and Numerical Simulation, vol. 10, no. 6, pp. 681-691, 2005.

[42] D. Jana, "Chaotic dynamics of a discrete predator-prey system with prey refuge," Applied Mathematics and Computation, vol. 224, pp. 848-865, 2013.

[43] M. Liao, X. Tang, and C. Xu, "Bifurcation analysis for a threespecies predator-prey system with two delays," Communications in Nonlinear Science and Numerical Simulation, vol. 17, no. 1, pp. 183-194, 2012.

[44] X.-Y. Meng, H.-F. Huo, and X.-B. Zhang, "Stability and global Hopf bifurcation in a delayed food web consisting of a prey and two predators," Communications in Nonlinear Science and Numerical Simulation, vol. 16, no. 11, pp. 4335-4348, 2011.

[45] G.-P. Hu, W.-T. Li, and X.-P. Yan, "Hopf bifurcations in a predator-prey system with multiple delays," Chaos, Solitons and Fractals, vol. 42, no. 2, pp. 1273-1285, 2009.

[46] Y. Song, Y. Peng, and J. Wei, "Bifurcations for a predator-prey system with two delays," Journal of Mathematical Analysis and Applications, vol. 337, no. 1, pp. 466-479, 2008.

[47] X.-P. Yan and Y.-D. Chu, "Stability and bifurcation analysis for a delayed Lotka-Volterra predator-prey system," Journal of Computational and Applied Mathematics, vol. 196, no. 1, pp. 198210, 2006.

[48] H. I. Freedman, Deterministic Mathematical Models in Population Ecology, HIFR Consulting, Edmonton, Canada, 1987.

[49] Y. Kuang, Delay Differential Equations with Applications in Population Dynamics, Academic Press, New York, NY, USA, 1993. 
[50] N. Bairagi and D. Jana, "On the stability and Hopf bifurcation of a delay-induced predator-prey system with habitat complexity," Applied Mathematical Modelling, vol. 35, no. 7, pp. 3255-3267, 2011.

[51] S. Ruan, "On nonlinear dynamics of predator-prey models with discrete delay," Mathematical Modelling of Natural Phenomena, vol. 4, no. 2, pp. 140-188, 2009.

[52] S. Ruan and J. Wei, "On the zeros of transcendental functions with applications to stability of delay differential equations with two delays," Dynamics of Continuous, Discrete and Impulsive Systems Series A: Mathematical Analysis, vol. 10, no. 6, pp. 863874, 2003.

[53] J. Hale, Theory of Functional Differential Equations, Springer, Berlin, Germany, 1977.

[54] S. Mandal, D. Jana, A. B. Roy, and N. C. Majee, "Chaotic behavior of a class of neural network with discrete delays," International Journal of Modern Nonlinear Theory and Application, vol. 2, no. 1A, pp. 97-101, 2013.

[55] S. H. Strogatz, Nonlinear Dynamics and Chaos: With Applications to Physics, Biology, Chemistry, and Engineering, Westview Press, 2009.

[56] K. Wang, W. Wang, H. Pang, and X. Liu, "Complex dynamic behavior in a viral model with delayed immune response," Physica D: Nonlinear Phenomena, vol. 226, no. 2, pp. 197-208, 2007.

[57] A. H. Nayfeh and B. Balachandran, Applied Nonlinear Dynamics, Wiley, New York, NY, USA, 1995.

[58] N. Marwan, M. Carmen Romano, M. Thiel, and J. Kurths, "Recurrence plots for the analysis of complex systems," Physics Reports, vol. 438, no. 5-6, pp. 237-329, 2007. 

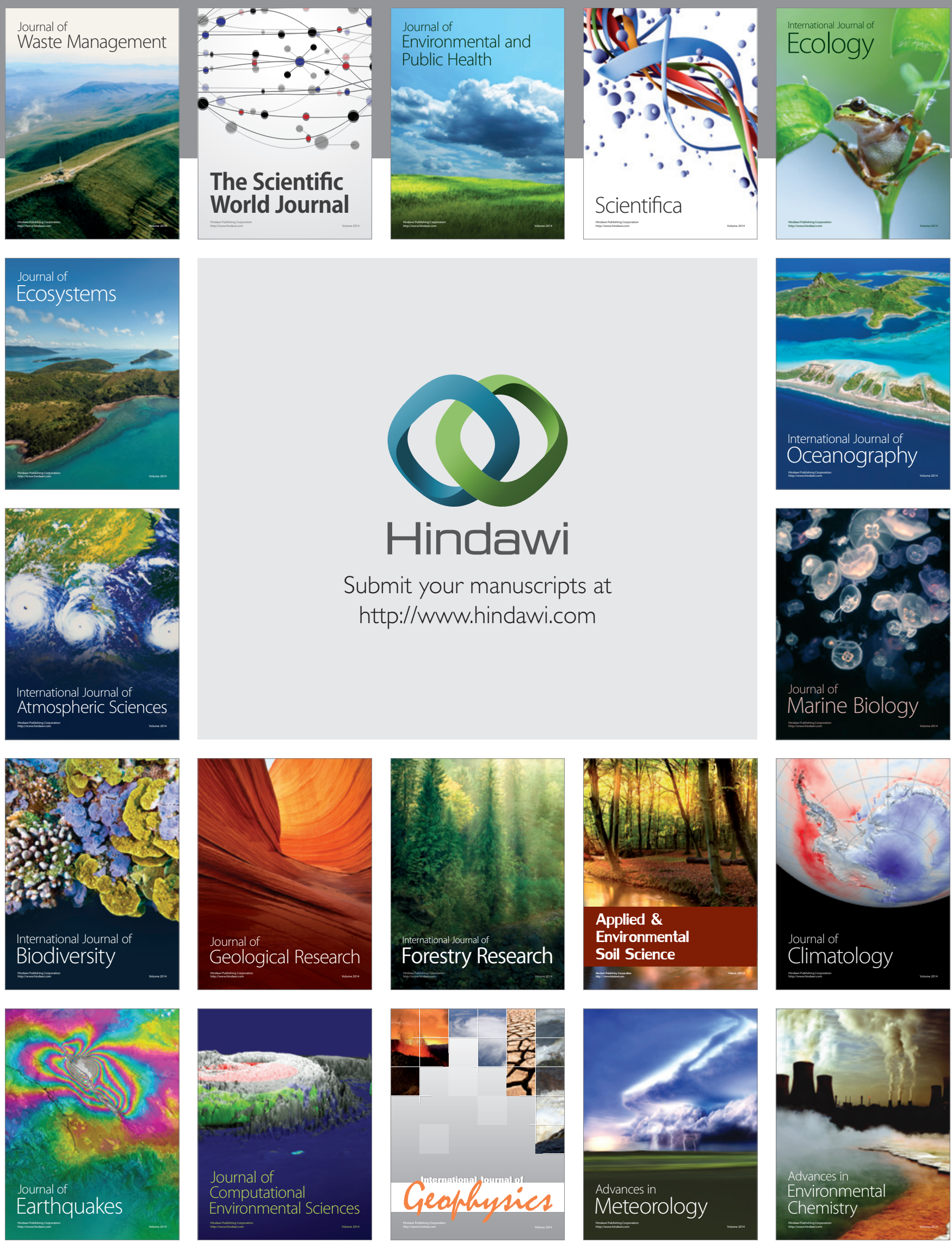